\title{
Scattering Functions of Core-Shell-Struct ured Hard Spheres with Schulz-Distributed Radii
}

\author{
M. Nayeri, ${ }^{\dagger}$ M. Zackrisson, ${ }^{\ddagger}$ and J. Bergenholtz ${ }^{*, \dagger}$ \\ Department of Chemistry, University of Gothenburg, SE-41296 Göteborg, Sweden, and Adolphe Merkle \\ Institute, University of Fribourg, Route de l'Ancienne Papeterie, P. O. Box 209, CH-1723 Marly 1, Switzerland
}

\begin{abstract}
The scattering intensity of polydisperse systems of core-shell and layered hard spheres is considered. The Percus-Yevick solution for the partial structure factors is cast in a form suitable for numerical and analytical treatment. Closed-form, analytical expressions are given for an effective hard-sphere model of the scattering intensity of particles with an internal layered structure and a size polydispersity governed by a Schulz distribution. A similar model for polydisperse hard spheres of core-shell structure but with a monodisperse shell thickness is also presented. The models are tested against small-angle X-ray scattering experiments on a hard-sphere-like microemulsion system.
\end{abstract}

\section{Introduction}

Small-angle scattering provides a means of microscopic characterization of a wide range of systems, and it is a powerful probe of structure and interactions in colloidal systems. ${ }^{1}$ Analysis of scattering results from disordered systems of colloidal particles often proceeds by making quantitative comparisons with a well-defined model, though in many cases modelindependent analyses can also be used very effectively. ${ }^{2-4}$ In the former case, however, models are defined at the outset in terms of a set of assumptions about, e.g., particle size, shape, and size distribution. This is a commonly used route to obtaining single-particle structural data on colloidal particles. ${ }^{5}$ However, for interacting systems analysis along these lines is more difficult. Even for spherical particles, polydispersity in particle size makes the disentangling of internal particle structure, size distribution, and interactions a complicated task.

Focusing on spherical particles with central, isotropic interactions, there are established routes within liquid-state theory for obtaining model results for the average scattering intensity. ${ }^{6,7}$ One common approach is to approximate the system by a fewcomponent, effective mixture ${ }^{8,9}$ (for a different approach, see Lado $^{10}$ ). The coupled Ornstein-Zernike integral equations can then be solved numerically, yielding the partial structure factors of the mixture for an approximate closure relation, interaction potential, and mixture composition. This prescription is very flexible in that it can be applied for essentially any interaction potential, size distribution, distribution of scattering contrast, and closure relation. ${ }^{7-9,11-13}$ In practice, however, full numerical solutions of this sort are usually too time-consuming, especially given that a rather large number of components is often required for modeling scattering data extending to wave vectors beyond the first correlation peak. Consequently one may want to turn to simpler approaches for analysis.

For a few closure/interaction potential combinations analytical solutions exist to the multicomponent integral equations. The most well-known is the analytical solution afforded by the

* To whom correspondence should be addressed. E-mail: jbergen@ chem.gu.se

University of Gothenburg.

* University of Fribourg.
Percus-Yevick closure for mixtures of hard-sphere particles. ${ }^{14,15}$ Vrij $^{16}$ and Blum and Stell ${ }^{17}$ in effect analytically inverted the matrix of direct correlation functions to obtain the partial structure factors needed to compute the scattering intensity. Their results, or those from other analytical expressions for partial structure factors, ${ }^{18-21}$ can be used with a given singleparticle form amplitude and a histogram representation of the size distribution. ${ }^{22}$ Again, many components may be required in the histogram representation to model polydisperse systems properly, and calculations, particularly if they are carried out within a least-squares minimization procedure, ${ }^{5}$ may become too time-consuming for routine use. As an alternative, one can make further simplifying assumptions. ${ }^{23-25}$ A different tack was taken by Griffith et al. ${ }^{26}$ who considered a continuous, Schulz size distribution and, on the basis of the Percus-Yevick theory, ${ }^{14-17}$ completed the required integrations to obtain an analytical expression for the scattering intensity. This analytical model makes for rapid evaluations of scattering intensities, but it is quite restricted in that it applies only to homogeneous hard spheres.

In what follows, we stay with the Percus-Yevick theory, which predicts accurate structure factors up to quite large volume fractions and polydispersities, ${ }^{27}$ and extend the hard-sphere model of Griffith et al. $^{26}$ in a number of ways. We consider two different layered distributions of scattering contrast, which requires altering the single-particle form amplitude. In both cases, a Schulz distribution is employed to handle size polydispersity and closed-form, analytical expressions are presented for the scattering intensity. Furthermore, in these models we introduce an effective interaction diameter that can be different from the diameter that contributes directly to the scattering intensity through the form amplitude. With these extensions a wider range of colloidal sphere systems can be modeled, including vesicles, composite particles of core-shell and layered internal structures, and spherical particles exhibiting more complex distributions of scattering contrast. We test the developed models on a well-characterized microemulsion system. 


\section{Experimental Section}

Materials. The nonionic surfactant pentaethylene glycol dodecyl ether $\left(\mathrm{C}_{12} \mathrm{E}_{5}\right)$ and $n$-decane were obtained from Nikko Chemicals and Sigma and were used as received. A microemulsion stock solution was prepared by weighing in $\mathrm{C}_{12} \mathrm{E}_{5}$, Milli-Q water, and $n$-decane, giving a surfactant-to-oil mass ratio of 1.08 , in agreement with Olsson and Schurtenberger, ${ }^{28}$ and a volume fraction $\phi_{\mathrm{o}+\mathrm{s}}=0.396$. Here, $\phi_{\mathrm{o}+\mathrm{s}}=\phi_{\mathrm{o}}+\phi_{\mathrm{s}}$ refers to the volume fraction of oil plus surfactant, which was determined from the mass-based composition and bulk component densities. ${ }^{29}$ Dilution of the stock solution with water yielded a concentration series comprising six samples in total. The emulsification-failure boundary, i.e., the temperature below which homogeneous microemulsions expel an excess oil phase, occurs around $25{ }^{\circ} \mathrm{C} .{ }^{30}$ As in the small-angle neutron scattering (SANS) study by Bagger-Jörgensen et al., ${ }^{31}$ the experiments were carried out a few degrees below this temperature, where samples are metastable; no phase separation was observed during the time of the measurements.

Small-Angle X-ray Scattering. Small-angle X-ray scattering (SAXS) measurements were conducted on beam line I711 at the MAX II storage ring in Lund, Sweden. ${ }^{32}$ Microemulsion samples were enclosed in high-quality quartz capillaries with a diameter of $1.7 \mathrm{~mm}$, glued in steel housings. A water sample, the solvent background, was measured in the same capillary and later subracted in the data treatment. Good statistics were accumulated within $600 \mathrm{~s}$ for the dilute samples and $300 \mathrm{~s}$ for the more concentrated ones. In the data reduction the beam decay and transmission of the samples were taken into account by measuring the beam intensity with a semiconducting diode moved into the beam immediately before and after sample exposure. The sample-to-detector distance was $1.5 \mathrm{~m}$, and the wavelength was $1.025 \AA$. The isotropic scattering data were azimuthally averaged, yielding one-dimensional data in terms of the modulus of the scattering vector $k=4 \pi \sin (\theta / 2) / \lambda$, where $\theta$ is the scattering angle and $\lambda$ is the wavelength. The excess scattering was determined by subtracting the background and correcting for transmissions and electronic noise. The experiments have not been converted to an absolute intensity scale, allowing for an arbitrary multiplicative shift. However, the spectra recorded for the different concentrations are internally consistent, and they are for this reason not shifted relative to one another.

Theory. For a continuous size distribution $f\left(\sigma_{\alpha}\right)$ the scattering intensity is given by ${ }^{17,26}$

$$
\begin{aligned}
I(k)= & I_{1}(k)+I_{2}(k)=n \int_{0}^{\infty} \mathrm{d} \sigma_{\alpha} f\left(\sigma_{\alpha}\right) F_{\alpha}{ }^{2}(k)+ \\
& n \int_{0}^{\infty} \mathrm{d} \sigma_{\alpha} \int_{0}^{\infty} \mathrm{d} \sigma_{\beta} f\left(\sigma_{\alpha}\right) f\left(\sigma_{\beta}\right) F_{\alpha}(k) F_{\beta}(k) H_{\alpha \beta}(k)
\end{aligned}
$$

where $n$ is the total number density of particles, $k$ is the modulus of the wave vector, $F_{\alpha}(k)$ is the single-particle form amplitude, and $H_{\alpha \beta}(k)=\left(n_{\alpha} n_{\beta}\right)^{1 / 2} h_{\alpha \beta}(k)$ are essentially the Fouriertransformed total correlation functions, related to the partial structure factors as $S_{\alpha \beta}(k)=\delta_{\alpha \beta}+H_{\alpha \beta}(k)$, with $n_{\alpha}$ being the number density of particles of size fraction $\alpha$. The $H_{\alpha \beta}(k)$ functions have a simple mathematical structure in the PercusYevick solution for hard spheres. ${ }^{16,17}$ In particular, the dependence on the particle diameters of the size distribution is such that the double integral in eq 1 can be factored into products of integrals. The integral $I_{2}(k)$ can be expressed compactly as

$$
I_{2}(k)=-\frac{2 n^{2} k^{-3}}{X^{2}+Y^{2}}\left(2 \mathscr{T}_{1}(k)+\mathscr{T}_{2}(k)-\mathscr{T}_{3}(k)\right)
$$

where the $\mathscr{T}_{i}(k)$ functions are double integrals, each of which factors as

$$
\begin{gathered}
\mathscr{T}_{1}(k)=M_{+}^{(1,6)} \mathcal{S}_{0}(k) \mathscr{O}_{0}(k)+M_{+}^{(2,4)}\left[\mathcal{S}_{1}(k) \mathscr{O}_{0}(k)+\right. \\
\left.\int_{0}(k) \mathscr{O}_{1}(k)\right]+M_{+}^{(3,5)} \mathcal{S}_{1}(k) \mathcal{O}_{1}(k) \\
\mathscr{T}_{2}(k)=M_{-}^{(6,1)} \mathcal{O}_{0}(k)^{2}+2 M_{-}^{(4,2)} \mathscr{C}_{0}(k) \mathscr{O}_{1}(k)+M_{-}^{(5,3)} \mathscr{C}_{1}(k)^{2} \\
\mathscr{T}_{3}(k)=M_{-}^{(6,1)} \mathcal{S}_{0}(k)^{2}+2 M_{-}^{(4,2)} \mathcal{S}_{0}(k) \mathcal{S}_{1}(k)+M_{-}^{(5,3)} \mathcal{S}_{1}(k)^{2}
\end{gathered}
$$

in terms of the basic integrals

$$
\begin{aligned}
& S_{n}(k)=\int_{0}^{\infty} \mathrm{d} \sigma_{\alpha} \sigma_{\alpha}{ }^{n} f\left(\sigma_{\alpha}\right) F_{\alpha}(k) \sin \left(\frac{k \sigma_{\alpha}}{2}\right) \\
& \mathcal{O}_{n}(k)=\int_{0}^{\infty} \mathrm{d} \sigma_{\alpha} \sigma_{\alpha}{ }^{n} f\left(\sigma_{\alpha}\right) F_{\alpha}(k) \cos \left(\frac{k \sigma_{\alpha}}{2}\right)
\end{aligned}
$$

with $n=0$ or 1 . In what follows, due to the factoring, we can dispense with the size indices $\alpha$ and $\beta$. The prefactors in the above are given as $M_{ \pm}^{(i, j)}=\delta_{j} Y \pm \delta_{i} X$, where $X, Y$, and $\delta_{1}-\delta_{6}$ are the same as those given by Griffith et al. ${ }^{26}$

We follow Griffith et al. ${ }^{26}$ and choose $f(\sigma)$ as a Schulz distribution, ${ }^{33-35}$ which is given by

$$
f(\sigma)=\frac{\sigma^{c-1}}{b^{c} \Gamma(c)} \mathrm{e}^{-\sigma / b}
$$

where $b=\bar{\sigma} / c, \Gamma(c)$ is the $\Gamma$ function of argument $c=z+1$, and $\bar{\sigma}$ is the mean diameter. As written, the distribution fulfills the normalization condition $\int_{0}^{\infty} \mathrm{d} \sigma f(\sigma)=1$. The parameter $z$ is related to the polydispersity in terms of the normalized standard deviation $s_{\sigma}$, viz., $s_{\sigma}=\left(\overline{\sigma^{2}} / \bar{\sigma}^{2}-1\right)^{1 / 2}=(z+1)^{-1 / 2}$. Griffith and co-workers integrated eq 1 analytically for homogeneous (Percus-Yevick) hard spheres with Schulz-distributed diameters. ${ }^{26}$ We shall extend their solution by adopting for $F(k)$ a distribution of scattering material comprising a spherical core surrounded by one or more concentric shells. In addition, we will introduce a diameter for the hard-sphere interaction that can be different from the one that enters the form amplitude. This leads to an effective hard-sphere model that may be used to model extra excluded-volume interactions.

Model A: Core-Multishell Spheres with Coupled Polydispersity. We assume that the distribution of scattering contrast in the spherical particles is partitioned in a core of radius $a_{1}=$ $p_{1} a_{N}$, followed by a sequence of $N-1$ concentric shells, each extending to an outer radius of $a_{i}=p_{i} a_{N}$ with $p_{N}=1$. We neglect any effects of fluctuations ${ }^{36,37}$ in the excess scattering contrast, e.g., electron or scattering length density, $\rho(r)-\rho_{\text {solv }}$, and give the contrast in each layer a constant value $\rho_{i}-\rho_{\text {solv }}$. Note that in this model, which we will refer to as model $\mathrm{A}$, the radii are proportional to one another through the constant $p_{i}$ factors. For this model the single-particle form amplitude can be expressed as $^{24}$

$$
F(k)=\frac{4 \pi}{k^{3}} \sum_{j=1}^{N} \rho_{j, j+1} y\left(\frac{k p_{j} \sigma}{2 p}\right)
$$

where $\rho_{j, j+1}=\rho_{j}-\rho_{j+1}, \rho_{N+1}=\rho_{\text {solv }}$, and $y(x)=\sin x-x \cos$ $x$. The parameter $p$ is introduced in eq 9 as an additional proportionality factor, viz., $p=\sigma / 2 a_{N}$; it is the ratio between the effective hard-sphere (interaction) diameter, $\sigma$, and the 
diameter entering the form amplitude, $2 a_{N}$. With this model we obtain the same normalized standard deviation, $(z+1)^{-1 / 2}$, regardless of whether we consider the size distribution as governing core, shell, or effective hard-sphere radii or diameters.

Substituting eqs 8 and 9 in eqs 6 and 7 leads to

$$
\begin{aligned}
& \int_{n}(k)=\frac{4 \pi}{k^{3}} \sum_{i=1}^{N} \rho_{i, i+1}\left[A_{n}\left(\frac{k p_{i}}{2 p}, \frac{k}{2}\right)-\frac{k p_{i}}{2 p} C_{n+1}\left(\frac{k}{2}, \frac{k p_{i}}{2 p}\right)\right] \\
& O_{n}(k)=\frac{4 \pi}{k^{3}} \sum_{i=1}^{N} \rho_{i, i+1}\left[C_{n}\left(\frac{k p_{i}}{2 p}, \frac{k}{2}\right)-\frac{k p_{i}}{2 p} B_{n+1}\left(\frac{k}{2}, \frac{k p_{i}}{2 p}\right)\right]
\end{aligned}
$$

where $A_{n}(k, q), B_{n}(k, q)$, and $O_{n}(k, q)$ are given by analytical expressions in the Appendix. With explicit expressions for the integrals in eqs 6 and 7 in hand, the calculation of $I_{2}(k)$ in eq 1 follows from evaluating eqs $2-5$. The first integral $I_{1}(k)$ remains to be determined. It is essentially the form factor $P(k)=I_{1}(k) /$ $n$. The form factor for a core-shell model with coupled polydispersity, model A with $F(k)$ as in eq 9 with $N=2$, was first determined by Hayter ${ }^{24}$ for Schulz-distributed radii, thereby generalizing Aragón and Pecora's solutions for homogeneous and hollow spheres. ${ }^{35}$ Hayter's result generalized to a core with $N-1$ shells reads as follows in the present notation:

$$
\begin{gathered}
I_{1}(k)=\left(\frac{4 \pi}{k^{3}}\right)^{2} n \sum_{i, j=1}^{N} \rho_{i, i+1} \rho_{j, j+1}\left[A_{0}\left(\frac{k p_{i}}{2 p}, \frac{k p_{j}}{2 p}\right)-\frac{k p_{i}}{2 p} C_{1}\left(\frac{k p_{j}}{2 p}, \frac{k p_{i}}{2 p}\right)-\right. \\
\left.\frac{k p_{j}}{2 p} C_{1}\left(\frac{k p_{i}}{2 p}, \frac{k p_{j}}{2 p}\right)+\frac{k p_{i}}{2 p} \frac{k p_{j}}{2 p} B_{2}\left(\frac{k p_{i}}{2 p}, \frac{k p_{j}}{2 p}\right)\right]
\end{gathered}
$$

Despite appearances, the expression is independent of $p$, which appears here only because the Schulz distribution has been assigned to govern the effective hard-sphere diameter.

Model B: Core-Shell Spheres with Uniform Shell Thickness. In model A, large particles are surrounded by shells with proportionately larger thicknesses than those around smaller particles. In many situations, however, e.g., vesicle dispersions and perhaps droplet microemulsions, it may be more physically motivated to use a model with a uniform shell thickness. Such a core-shell model was considered by Bartlett and Ottewill, who derived the form factor for polydisperse particles with a constant shell thickness, ${ }^{38}$ and Yan and Clarke, who considered the forward scattering $(k \rightarrow 0)$ limit. ${ }^{39}$ However, introducing hard-sphere, structure-factor effects in this model requires care. To ensure that the distribution does not include core radii, here labeled $a$, with negative values, we take the Schulz distribution to govern the core radius rather than the hard-sphere diameter. It follows that $f(a)=a^{c-1} b^{-c} \mathrm{e}^{-a / b} / \Gamma(c)$ with $b=\bar{a} / c$ and $c=z$ +1 . The Schulz parameter $z$ is now set by the normalized standard deviation of the core radius distribution; i.e., $z+1=$ $1 / s_{a}^{2}=\left(\overline{a^{2}} / \bar{a}^{2}-1\right)^{-1}$. For simplicity we only consider particles composed of a core with a single shell. In keeping with the effective hard-sphere model, we set the interaction diameter as $\sigma=2 p(a+\delta)$, where $\delta$ is the shell thickness and $p$ is the ratio between the hard-sphere and actual diameters. In this model, which we refer to henceforth as model $\mathrm{B}, \delta$ and $p$ are kept constant.

For particles with the contrast partitioned in a core and a single shell, the form amplitude is given as $F(k)=$ $4 k \pi^{-3}\left(\rho_{12} y\left(k a_{1}\right)+\rho_{23} y\left(k a_{2}\right)\right)$. We set $a_{1}=a$ and $a_{2}=a+\delta$, and, in anticipation of integrations with respect to $a$, we decompose $y(k a+k \delta)$ leading to

$$
\begin{array}{r}
F(k)=\frac{4 \pi}{k^{3}}\left[\omega_{1} \sin (k a)+\omega_{2} \cos (k a)+\omega_{3} k a \sin (k a)+\right. \\
\left.\omega_{4} k a \cos (k a)\right]
\end{array}
$$

where

$$
\begin{gathered}
\omega_{1}=\rho_{12}+\rho_{23}(\cos (k \delta)+k \delta \sin (k \delta)) \\
\omega_{2}=\rho_{23}(\sin (k \delta)-k \delta \cos (k \delta)) \\
\omega_{3}=\rho_{23} \sin (k \delta) \\
\omega_{4}=-\rho_{12}-\rho_{23} \cos (k \delta)
\end{gathered}
$$

For model A many results could be directly transcribed from the work of Griffith et al. ${ }^{26}$ This holds but to a lesser extent for model B. As summarized in the Appendix, an equivalent set of equations to eqs $2-7$ can be derived for a size distribution governing the core radius when it is tied to the hard-sphere diameter as $\sigma=2 p(a+\delta)$. In the Appendix we also provide expressions for the remaining parameters needed to evaluate $I_{1}(k)$ and $I_{2}(k)$ for this model.

\section{Results and Discussion}

We begin by showing some results of the models, focusing on the extensions with respect to the scattering function for polydisperse, homogeneous spheres as worked out by Griffith et al. ${ }^{26}$ In the models defined here, the particles need not act as true hard spheres in the sense that they can be given a larger hard-sphere diameter than the diameter that contributes directly to the scattering through the form amplitude. This effect is controlled by the parameter $p$, the ratio of the interaction diameter to the actual diameter, and is best illustrated at low particle concentrations. For simplicity we use model A with homogeneous $(N=1)$ particles and define $\phi_{a}=4 n \pi \overline{a_{1}^{3}} / 3$ and $\phi_{\sigma}=4 n \pi p^{3} \overline{a_{1}^{3}} / 3$. In Figure 1 some results for so-called measured or effective structure factors, ${ }^{40} S^{\mathrm{M}}(k)=(n P(k))^{-1} I(k)$, are shown. The left panel shows results for true $(p=1)$ hard spheres of mean radius $\bar{a}_{1}$ as the number density increases. The right panel shows effective hard spheres of the same mean radius $\bar{a}_{1}$ at

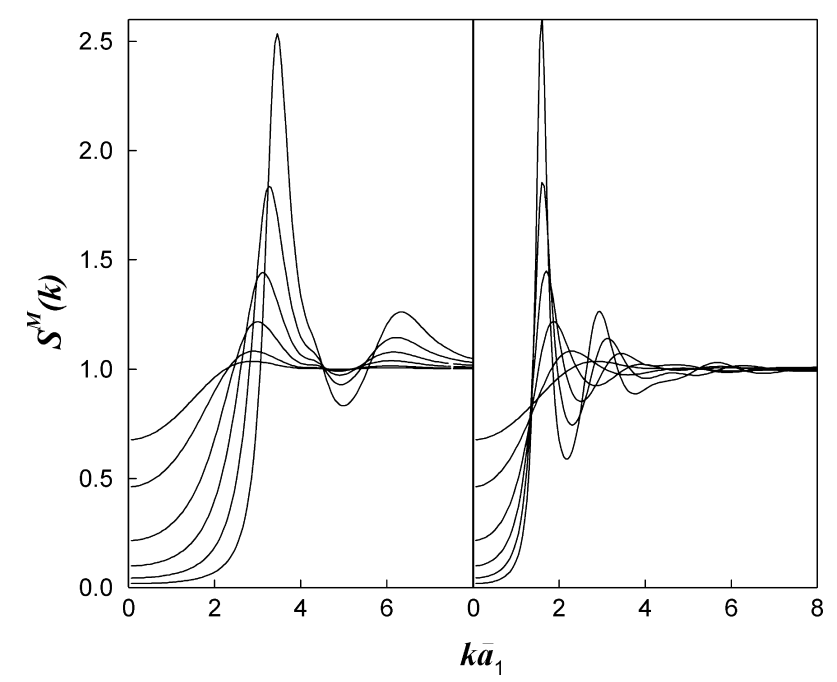

Figure 1. Measured structure factors as functions of $k \bar{a}_{1}$ for homogeneous $(N=1)$ spheres using model A with a polydispersity, $s_{\sigma}$, equal to 0.1 . The left panel shows the effect of increasing volume fraction; from top-to-bottom along the left-hand side of the panel, $\phi_{a}=0.05$, $0.1,0.2,0.3,0.4$, and 0.5 at $p=1$. The right panel shows results for $\phi_{a}=0.05$ and, from top-to-bottom along the left-hand side of the panel, $p=1,1.26,1.587,1.817,2.00$, and 2.154. For this set of $p$ values $\phi_{\sigma}$ $=p^{3} \phi_{a} \approx 0.05,0.1,0.2,0.3,0.4$, and 0.5 . 


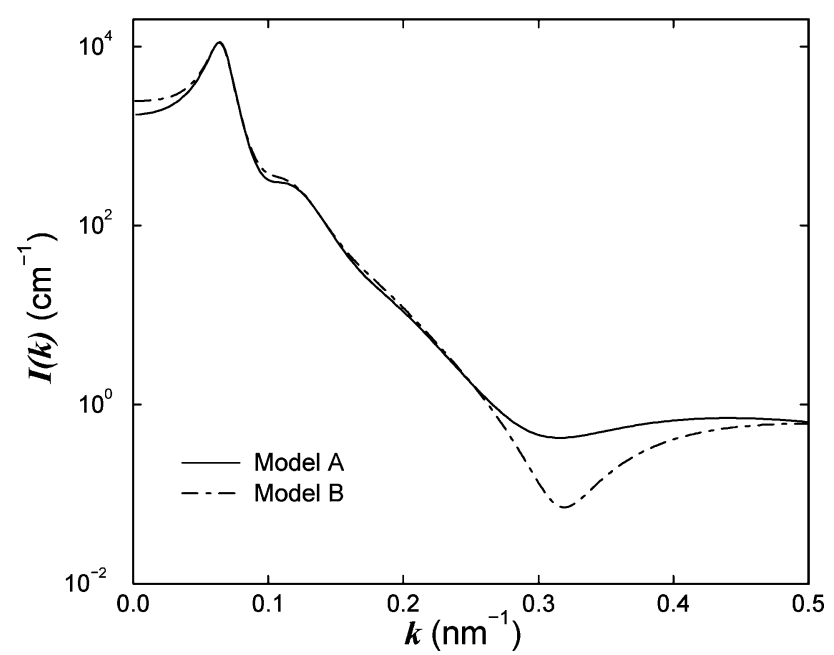

Figure 2. Scattered intensity as a function of $k$ for core - shell $(N=$ 2) particles using models $\mathrm{A}$ and B with $\phi_{\sigma}=0.5, s_{\sigma}=0.15, p=1, \bar{a}_{2}$ $=50 \mathrm{~nm}, \rho_{13}=2 \times 10^{-4} \mathrm{~nm}^{-2}$, and $\rho_{23}=1 \times 10^{-4} \mathrm{~nm}^{-2}$. Also, in model A $p_{1}=0.8$ and in model $\mathrm{B} \delta=10 \mathrm{~nm}$, giving the same average core and total radius in both cases. Note, however, that the polydispersity with respect to the core radius is $15 \%$ in model $\mathrm{A}$, whereas it becomes $18.75 \%$ for model B.

constant number density as $p$ increases; i.e., $\phi_{a}$ remains constant, while $\phi_{\sigma}$ increases. In the former case, model A reduces to the result of Griffith et al., ${ }^{26}$ whereas the latter could account for the presence of some extra repulsion among particles. The concentrations and effective hard-sphere diameters in the comparison have been chosen so that $\phi_{\sigma}=p^{3} \phi_{a}$, which yields structure factors of similar magnitude. As seen in Figure 1, whereas increasing concentration leads to a primary structure factor peak shifting toward higher $k$ for true hard spheres, the peak shifts to smaller $k$ as the hard-sphere diameter is inflated at constant number density. Note that comparing the structure factors as a function of $k \sigma$ instead of $k$ or $k \bar{a}_{1}$ would have resulted in agreement at low $k$ but differences at large $k$. This can be understood by the polydispersity and the third moment $n \overline{\sigma^{3}}$ being the same in this particular comparison. Equating the first few moments typically works well for reproducing low- $k$ features of static scattering and is often sufficient to capture the main correlation peak in terms of height and position, ${ }^{9}$ but differences among higher order moments cause disagreement at larger $k$.

In Figure 2 we illustrate the main difference between models $\mathrm{A}$ and $\mathrm{B}$, here in terms of the intensity of a concentrated core-shell $(N=2)$ system. In model A the shell thickness is polydisperse, whereas in model B the shell thickness is uniform. The average core radius in model $\mathrm{A}$ is $\bar{a}_{1}=p_{1} \bar{a}_{2}$, and in model $\mathrm{B}$ it is $\bar{a}_{1}=\bar{a}_{2}-\delta$. Clearly, in model $\mathrm{B}$, had a Schulz distribution been assigned to govern $a_{2}$ one could not have guaranteed a corresponding distribution of positive-semidefinite $a_{1}$ values. In the comparison in Figure 2 between models $\mathrm{A}$ and $\mathrm{B}$, the mean core radius has been set to the same value, 40 $\mathrm{nm}$, and the mean core-shell radius $\bar{a}_{2}$ has also been set to the same value, $50 \mathrm{~nm}$. As seen, differences between the models appear primarily at large $k$, where model B with a monodisperse shell thickness produces more pronounced oscillations compared to model A with polydisperse shells. The differences between the models at low $k$ come about because setting the same average core and total radii in the two models leads necessarily to differences in polydispersity. In this case, the polydispersity with respect to the hard-sphere diameter has been equated between the two models at $15 \%$, which leads to a larger

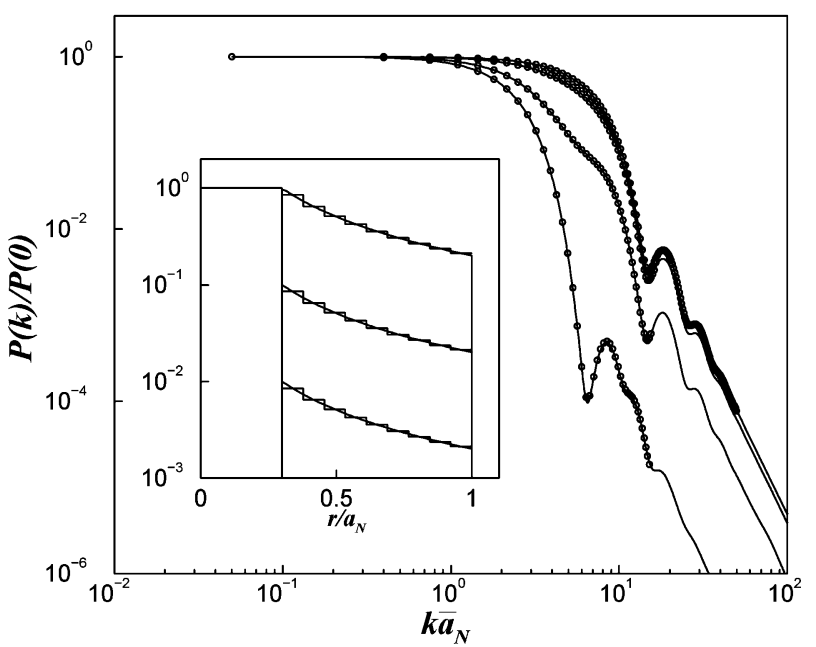

Figure 3. Normalized form factor as a function of $k \bar{a}_{N}$ for model A with a $N=10$ discretization approximation of contrast profiles given by $\rho_{\text {shell }}(r)-\rho_{\text {solv }}=\Delta \rho\left(r / a_{1}\right)^{-4 / 3}$, with $\Delta \rho=0,0.01,0.1$, and 1 , as shown in the inset, and $a_{1} / a_{N}=p_{1}=0.3$. All form factors have been determined for a polydispersity of $10 \%$. For reference, form factors corresponding to the nondiscretized contrast profiles are shown using symbols. The result for homogeneous spheres $(\Delta \rho=0)$ is, proceeding along the right-hand side of the main panel, the top curve. The results for $\Delta \rho=0.01,0.1$, and 1 follow in descending order along the righthand side.

polydispersity for the core radius of model B. As seen in Figure 2 , in going from the results of model A to model B, this leads precisely to what one generally observes in connection with an increase in size polydispersity: ${ }^{8,40,41}$ enhanced scattering at the lowest $k$, a reduction of the main correlation peak (hardly visible in Figure 2), and a smearing of the first form factor oscillation at $k \approx 0.1 \mathrm{~nm}^{-1}$.

With a multilayer model in hand one can readily generate scattering predictions for complex contrast profiles that do not themselves permit for analytical treatment even at the form factor level. As an illustration, we consider a homogeneous core surrounded by a single layer with an excess contrast varying with distance $r$ from the particle center as $\rho(r)-\rho_{\text {solv }}=$ $\Delta \rho\left(r / a_{1}\right)^{-4 / 3}$, where $a_{1}$ is the radius of the homogeneous core. This shell contrast profile can be relevant to dispersions of spherical particles bearing grafted polymer. ${ }^{42}$ Power-law contrast profiles have been studied by Förster and Burger, ${ }^{43}$ who developed a semianalytical scheme for evaluating the form factor. As an alternative, in Figure 3, we use model A with $N$ $=10$ and, as shown in the inset to Figure 3, divide the contrast profile in a core with nine additional layers to approximate the contrast in the shell. Figure 3 shows form factors corresponding to varying overall shell contrast, all calculated for a polydispersity of $10 \%$. For weakly scattering shells the form factor exhibits an oscillation with a minimum close to $k \approx 4.5 / \bar{a}_{1}$, which corresponds to the usual form factor minimum for homogeneous spheres. As the contrast of the shell is increased a new oscillation appears, which develops into a minimum for $\Delta \rho=1$, this time at $k \approx 6.5 / \bar{a}_{N}$. It follows that the particles appear "smaller" than homogeneous spheres of radius $\bar{a}_{N}$. For reference, in Figure 3, we compare with form factors for spheres surrounded by shells with a nondiscretized, $\Delta \rho\left(r / a_{1}\right)^{-4 / 3}$ contrast profile, computed essentially using the ascending (in $k$ ) part of the series expansion developed by Förster and Burger. ${ }^{43}$ As seen, using a sufficient number of layers, we can accurately reproduce the correct form factor. However, the discrete nature of the model is revealed by extra oscillations setting in roughly at $k$ $\approx 2 \pi / \Delta r$, where $\Delta r$ is the increment used for the discretization 


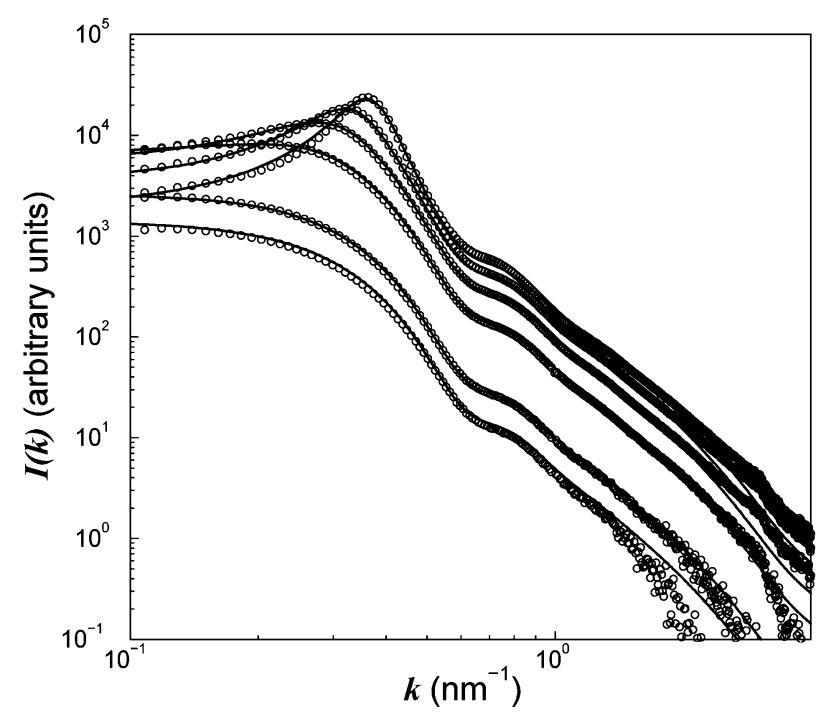

Figure 4. SAXS intensity as a function of wave vector for a concentration series of $\mathrm{C}_{12} \mathrm{E}_{5}$-based microemulsions of oil + surfactant volume fractions, from bottom-to-top along the right-hand side, $\phi_{\mathrm{o}+\mathrm{s}}$ $=0.0099,0.0198,0.0989,0.198,0.297$, and 0.396 . For clarity, only every other experimental $I(k)$ value is shown. The lines are fits to the data using model A (with $N=2$ ) as described in the text.

of the contrast profile. Hence, these oscillations are pushed beyond the $k$-window of interest, as done in Figure 3, by selecting a sufficiently fine resolution $\Delta r$. Hard-sphere structure factor effects can be added in Figure 3 as done in previous examples by including $I_{2}(q)$ in eq 2 .

We test the models on a well-characterized droplet-structured $\left(\mathrm{L}_{1}\right)$ microemulsion, comprising decane droplets stabilized by the nonionic surfactant $\mathrm{C}_{12} \mathrm{E}_{5}$ dissolved in water. The properties of the system have been well-captured by effective hard-sphere models for a surfactant-to-oil mass ratio of 1.08 at a temperature of $\approx 25^{\circ} \mathrm{C}$, when the system is close to expelling an excess oil phase (emulsification failure)..$^{28,31,44-46}$ Given that it is difficult to distinguish by small-angle scattering between polydisperse spheres and spheroidal particles, ${ }^{24,47}$ we proceed with modeling this system as composed of spherically shaped particles using models A and B. Indeed, along the emulsification-failure phase boundary, previous SANS experiments have shown that a model based on spherical particles can be used, at least to describe the large- $k$ scattering, and that the droplets maintain their size as a function of droplet concentration. ${ }^{31}$ Also, for the scattering of X-rays, the excess electron density of the shell is larger than that of the core for this system. ${ }^{48}$
In the modeling we have used a core with an electron density $\rho_{1}=253 \mathrm{e} / \mathrm{nm}^{3}$, a shell with an electron density $\rho_{2}$, to be determined, while the solvent electron density has been kept constant at $\rho_{3}=333 \mathrm{e} / \mathrm{nm}^{3}$. Similar to findings from the SANS study by Bagger-Jörgensen et al., ${ }^{31}$ the SAXS spectra in Figure 4 superpose at large $k$ when scaled by the volume fraction, $\phi_{0+s}$ $=\phi_{\mathrm{o}}+\phi_{\mathrm{s}}$, of oil and surfactant. An exception is noted in the region near the first form factor oscillation at the highest concentrations, where there is some enhanced intensity relative to $n P(k)$, suggesting that the data here are affected by structure factor effects. As a consequence of this observation, we look for a droplet radius and size polydispersity that do not vary as a function of concentration and opt for a global "best fit" to the whole concentration series using the hard-sphere models. The core radius and the polydispersity are determined by the location and degree of smearing of the form factor oscillation at lowto-moderate $\phi_{0+s}$. There is essentially no leeway in assigning values of $\bar{a}_{1}=7.0 \mathrm{~nm}$ and $s_{\sigma}=0.19$, independent of concentration. The former is in good agreement with the value reported by Bagger-Jörgensen and co-workers, whereas the latter is somewhat larger than the corresponding value from the same SANS study.$^{31}$ Furthermore, dealing with the entire data set, which must accommodate the known dilution factors used to prepare the samples from the stock solution, rapidly eliminates model $\mathrm{B}$, which does not reproduce the structure factor peak and the low- $k$ scattering of the experiments at the higher concentrations. Turning to model A, we initially neglect hydration of the droplets and enforce, $\phi_{0+s}=4 n \pi \overline{a_{2}} / 3$, which leads to quite reasonable fits to the experimental data over the whole concentration interval using $p=1.065$ independent of droplet concentration. There is some mismatch in the region of the first form factor oscillation at the two highest concentrations, where the model underpredicts the experimental intensity. Nevertheless, this procedure leads to the conclusion that reproducing the structure factor peak position of the experiment using model A requires that the core radius be placed according to $\phi_{\mathrm{o}}+0.25 \phi_{\mathrm{s}} \approx 4 n \pi p_{1}{ }^{3} \overline{a_{2}} / 3$, where the 0.25 factor obtained is independent of the droplet concentration. In contrast, enforcing at the outset a factor of, e.g., 0.5, ${ }^{31}$ the experimental peak position at high concentration cannot be reproduced with model A. Indeed, similar SAXS spectra and SANS results have been interpreted on the basis of models of interacting ellipsoidal particles. ${ }^{48,49}$

Although the fits of the model to the data are quite reasonable, further improvement is possible by allowing for some hydration of the shells surrounding the droplets. This implies that the condition $\phi_{0+\mathrm{s}}=4 n \pi \overline{a_{2}} / 3$ is relaxed and optimization of the

TABLE 1: Integrals and Their Notation for a Schulz Distribution Governing the Core Radius with $b=\bar{a} / c$ and $c=z+1=$ $s_{a}{ }^{-2}$ and with a Hard-Sphere Diameter Defined as $\sigma=2 p(a+\delta)^{a}$

\begin{tabular}{|c|c|c|c|}
\hline$g(\sigma)$ & $n$ & $\int_{0}^{\infty} \mathrm{d} a f(a) \sigma^{n} g(\sigma)$ & notation \\
\hline 1 & 1 & $2 p(b c+\delta)$ & $\xi^{\prime}$ \\
\hline 1 & 2 & $(2 p)^{2}\left(b^{2} c(c+1)+2 b c \delta+\delta^{2}\right)$ & $\xi^{\prime \prime}$ \\
\hline 1 & 3 & $(2 p)^{3}\left(b^{3} c(c+1)(c+2)+3 b^{2} c(c+1) \delta+3 b c \delta^{2}+\delta^{3}\right)$ & $\xi^{\prime \prime \prime \prime}$ \\
\hline $\sin (k \sigma)$ & 0 & $v_{1}^{c / 2} \sin \left(2 p k \delta+c \tan ^{-1}(2 p b k)\right)$ & $\psi$ \\
\hline $\sin (k \sigma)$ & 1 & $2 p b c v_{1}^{(c+1) / 2} \sin \left(2 p k \delta+(c+1) \tan ^{-1}(2 p b k)\right)+2 p \psi \delta$ & $\psi^{\prime}$ \\
\hline $\sin (k \sigma)$ & 2 & $\begin{array}{l}(2 p b)^{2} c(c+1) v_{1}^{(c+2) / 2} \sin \left(2 p k \delta+(c+2) \tan ^{-1}(2 p b k)\right)- \\
(2 p \delta)^{2} \psi+4 p \psi^{\prime} \delta\end{array}$ & $\psi^{\prime \prime}$ \\
\hline $\cos (k \sigma)$ & 0 & $v_{1}^{c / 2} \cos \left(2 p k \delta+c \tan ^{-1}(2 p b k)\right)$ & $\chi$ \\
\hline $\cos (k \sigma)$ & 1 & $2 p b c v_{1}^{(c+1) / 2} \cos \left(2 p k \delta+(c+1) \tan ^{-1}(2 p b k)\right)+2 p \chi \delta$ & $\chi^{\prime}$ \\
\hline $\cos (k \sigma)$ & 2 & $\begin{array}{l}(2 p b)^{2} c(c+1) v_{1}^{(c+2) / 2} \cos \left(2 p k \delta+(c+2) \tan ^{-1}(2 p b k)\right)- \\
(2 p \delta)^{2} \chi+4 p \chi^{\prime} \delta\end{array}$ & $\chi^{\prime \prime}$ \\
\hline
\end{tabular}

${ }^{a}$ Here, $s_{a}$ is the normalized standard deviation of the core radius distribution. Also, the $v_{1}$ parameter in the table is given by $v_{1}=(1+$ $\left.(2 p b k)^{2}\right)^{-1}$ and $\xi_{1}=n \zeta^{\prime}, \xi_{2}=n \zeta^{\prime \prime}$, and $\xi_{3}=n \zeta^{\prime \prime \prime}$. 
parameters now improves the agreement and leads to a larger hard-sphere volume fraction than $\phi_{\mathrm{o}+\mathrm{s}}$, which corresponds to a hard-sphere radius $p \bar{a}_{2} \approx 8.5 \mathrm{~nm}$ in agreement with values reported in the literature. ${ }^{28,31}$ This procedure also leads to $p \approx$ 1 ; in other words, from the standpoint of the results of model A, as shown in Figure 4, the droplets behave essentially as true hard spheres. However. in keeping with previous definitions of effective hard-sphere models, we can calculate $\left(\phi_{\sigma} / \phi_{\mathrm{o}+\mathrm{s}}\right)^{1 / 3}=$ 1.065 , suggesting quite some hydration of the droplets $(\approx 2.2$ water molecules per ethylene oxide segment of the surfactant headgroup) given that with $p=1$ we have $\sigma=2 a_{2}$. This value is somewhat larger than the $\approx 1.045$ often found by comparing experiment with hard-sphere theory, ${ }^{28,31,46}$ however, these comparisons have relied on monodipserse hard-sphere theory. Using the radii and polydispersity from the fits to the data we can also estimate a hydrodynamic radius, using ${ }^{41} \overline{a_{2}{ }^{6}} / \overline{a_{2}{ }^{5}}$, as $\approx 9.98 \mathrm{~nm}$, which is in good agreement with results from dynamic light scattering measurements at low volume fractions. ${ }^{28,46}$ Finally, the area per surfactant headgroup at the average location of the polar/apolar interface, as set by $\phi_{\mathrm{o}}+0.5 \phi_{\mathrm{s}},{ }^{31}$ is determined as $0.45 \mathrm{~nm}^{2}$, which is only slightly smaller than values found by Olsson and co-workers. ${ }^{30,31}$

\section{Conclusions}

Analytical scattering functions for core-shell and layered (effective) hard spheres, with core radii and diameters obeying a unimodal Schulz distribution, have been given. This opens for the possibility of modeling the intensity of polydisperse, hard-sphere-like particles of complex internal morphology with the Griffith et al. solution for homogeneous hard spheres as a limiting case. ${ }^{26}$ As an example, the small-angle X-ray scattering from microemulsion droplets containing nonionic surfactant is shown to be well-modeled as originating from a hard-sphere system of core-shell particles not only at low concentrations but up to rather high concentrations.

Acknowledgment. Financial support by the Swedish Foundation for Strategic Research (SSF) and the Swedish Research Council is gratefully acknowledged. J.B. is a Royal Swedish Academy of Sciences Research Fellow, supported by a grant from the Knut and Alice Wallenberg Foundation. We thank Mehran Asad Ayoubi for assistance during the SAXS measurements and Yngve Cerenius and MAX-lab at Lund University for granting access to the $\mathrm{I} 711$ beam line facility.

\section{Appendix}

We require slight generalizations of the integrals that appear in Griffith et al. ${ }^{26}$ These are readily arrived at by adding and subtracting tabulated integrals. ${ }^{50}$ Introducing the notation

$$
\begin{aligned}
& A_{n}(k, q)=\int_{0}^{\infty} \mathrm{d} \sigma \sigma^{n} f(\sigma) \sin (k \sigma) \sin (q \sigma) \\
& B_{n}(k, q)=\int_{0}^{\infty} \mathrm{d} \sigma \sigma^{n} f(\sigma) \cos (k \sigma) \cos (q \sigma) \\
& C_{n}(k, q)=\int_{0}^{\infty} \mathrm{d} \sigma \sigma^{n} f(\sigma) \sin (k \sigma) \cos (q \sigma)
\end{aligned}
$$

where $f(\sigma)$ is the normalized Schulz distribution in eq 8 , we obtain

$$
\begin{array}{r}
A_{n}(k, q)=-\frac{1}{2} b^{n} \frac{\Gamma(c+n)}{\Gamma(c)}\left\{\left[1+u_{+}^{2}\right]^{-(c+n) / 2} \cos v_{+}-\right. \\
\left.\left[1+u_{-}^{2}\right]^{-(c+n) / 2} \cos v_{-}\right\}
\end{array}
$$

$$
\begin{array}{r}
B_{n}(k, q)=\frac{1}{2} b^{n} \frac{\Gamma(c+n)}{\Gamma(c)}\left\{\left[1+u_{+}{ }^{2}\right]^{-(c+n) / 2} \cos v_{+}+\right. \\
\left.\left[1+u_{-}{ }^{2}\right]^{-(c+n) / 2} \cos v_{-}\right\} \\
C_{n}(k, q)=\frac{1}{2} b^{n} \frac{\Gamma(c+n)}{\Gamma(c)}\left\{\left[1+u_{+}{ }^{2}\right]^{-(c+n) / 2} \sin v_{+}+\right. \\
\left.\left[1+u_{-}{ }^{2}\right]^{-(c+n) / 2} \sin v_{-}\right\}
\end{array}
$$

where $b$ and $c$ are parameters of the Schulz distribution, $u_{ \pm}=$ $b(k \pm q)$, and $v_{ \pm}=(c+n) \tan ^{-1} u_{ \pm}$. Moreover, only terms with $n=0,1$, and 2 are needed, for which the following holds: $\Gamma(c+n) / \Gamma(c)=1, c, c(c+1)$ for $n=0,1$, and 2, respectively.

For model B, i.e., for particles with polydisperse cores surrounded by shells of constant thickness, the form amplitude is given by eq 13. The polydisperse form factor for this model has been determined by Bartlett and Ottewill for radii obeying a Schulz distribution. ${ }^{38}$ In the present notation their result reads

$$
\begin{gathered}
I_{1}(k)=\left(\frac{4 \pi}{k^{3}}\right)^{2} n\left[\omega_{1}{ }^{2} A_{0}(k, k)+2 \omega_{1} \omega_{2} C_{0}(k, k)+\right. \\
2 \omega_{1} \omega_{3} k A_{1}(k, k)+2 \omega_{1} \omega_{4} k C_{1}(k, k)+\omega_{2}{ }^{2} B_{0}(k, k)+ \\
\left.2 \omega_{2} \omega_{3} k C_{1}(k, k)+2 \omega_{2} \omega_{4} k B_{1}(k, k)+\omega_{3}{ }^{2} k^{2} A_{2}(k, k)\right)+ \\
\left.2 \omega_{3} \omega_{4} k^{2} C_{2}(k, k)+\omega_{4}{ }^{2} k^{2} B_{2}(k, k)\right]
\end{gathered}
$$

To include structure factor effects in this model requires some care. In this case eqs $2-5$ still hold but now with a slightly different meaning for the basic integrals

$$
\begin{aligned}
& \mathcal{S}_{n}(k)=\int_{0}^{\infty} \mathrm{d} a a^{n} f(a) F(k) \sin (k p a) \\
&=\frac{4 \pi}{k^{3}}\left[\omega_{1} A_{n}(k, k p)+\omega_{2} C_{n}(k p, k)+\right. \\
&\left.\omega_{3} k A_{n+1}(k, k p)+\omega_{4} k C_{n+1}(k p, k)\right] \\
& \mathscr{O}_{n}(k)=\int_{0}^{\infty} \mathrm{d} a a^{n} f(a) F(k) \cos (k p a) \\
&=\frac{4 \pi}{k^{3}}\left[\omega_{1} C_{n}(k, k p)+\omega B_{n}(k, k p)+\right. \\
&\left.\omega_{3} k C_{n+1}(k, k p)+\omega_{4} k B_{n+1}(k, k p)\right]
\end{aligned}
$$

where the integrations now extend over the core radius distribution. In addition, the prefactors in eqs $3-5$ are given by expressions analogous to those for model A, $M_{ \pm}^{(i j)}=\delta_{j}^{\prime} Y \pm \delta_{i}^{\prime} X$, but where the $\delta_{i}^{\prime}$ functions are given by

$$
\begin{aligned}
& \delta_{1}^{\prime}=\left(\delta_{1}+4 p \delta \delta_{2}+4 p^{2} \delta^{2} \delta_{3}\right) \cos (2 k p \delta)+\left(\delta_{6}+\right. \\
& \left.4 p \delta \delta_{4}+4 p^{2} \delta^{2} \delta_{5}\right) \sin (2 k p \delta) \\
& \delta_{2}^{\prime}=2 p\left(\delta_{2}+2 p \delta \delta_{3}\right) \cos (2 k p \delta)+2 p\left(\delta_{4}+\right. \\
& \left.2 p \delta \delta_{5}\right) \sin (2 k p \delta) \\
& \delta_{3}^{\prime}=(2 p)^{2}\left(\delta_{3} \cos (2 k p \delta)+\delta_{5} \sin (2 k p \delta)\right) \\
& \delta_{4}^{\prime}=2 p\left(\delta_{4}+2 p \delta \delta_{5}\right) \cos (2 k p \delta)-2 p\left(\delta_{2}+\right. \\
& \left.2 p \delta \delta_{3}\right) \sin (2 k p \delta) \\
& \delta_{5}^{\prime}=(2 p)^{2}\left(\delta_{5} \cos (2 k p \delta)-\delta_{3} \sin (2 k p \delta)\right) \\
& \delta_{6}^{\prime}=\left(\delta_{6}+4 p \delta \delta_{4}+4 p^{2} \delta^{2} \delta_{5}\right) \cos (2 k p \delta)-\left(\delta_{1}+\right. \\
& \left.4 p \delta \delta_{2}+4 p^{2} \delta^{2} \delta_{3}\right) \sin (2 k p \delta)
\end{aligned}
$$

in terms of the same $\delta_{i}$ functions that enter model A, and which are given explicitly by Griffith et al. ${ }^{26}$ The functions $X$ and $Y$ are given by the same expressions as in model A, and they depend on a number of functions that are given by Griffith et al. ${ }^{26}$ These functions, however, are defined somewhat differently 
for model B and consequently assume expressions different from model $\mathrm{A}$ and as quoted by Griffith et al. ${ }^{26}$ For model B the required functions are integrals of the general form $\int_{0}^{\infty} \mathrm{d} a$ $f(a) \sigma^{n} g(\sigma)$, and they are listed in Table 1 for Schulz-distributed core radii with $\sigma=2 p(a+\delta)$.

\section{References and Notes}

(1) Neutrons, X-rays and light scattering methods applied to soft condensed matter; Lindner, P., Zemb, Th., Eds.; North-Holland: Amsterdam, 2002. 3086.

(2) Warr, G. G.; Zemb, T. N.; Drifford, M. J. Phys. Chem. 1990, 94,

(3) Pedersen, J. S. Curr. Opin. Colloid Interface Sci. 1999, 4, 190.

(4) Fritz, G.; Glatter, O. J. Phys.: Condens. Matter 2006, 18, S2403.

(5) Pedersen, J. S. Adv. Colloid Interface Sci. 1997, 70, 171

(6) Hansen, J.-P.; McDonald, I. R. Theory of Simple Liquids, 2nd ed.; Academic Press: San Diego, 1986.

(7) Klein, R.; D'Aguanno, B. In Light scattering, principles and development; Brown, W., Ed.; Oxford Science: Oxford, U.K., 1996; pp $30-102$.

(8) D’Aguanno, B.; Klein, R. J. Chem. Soc., Faraday Trans. 1991, 87,379

(9) D’Aguanno, B.; Klein, R. Phys. Rev. A 1992, 46, 7652.

(10) Lado, F. Phys. Rev. E 1996, 54, 4411.

(11) Krause, R.; D’ Aguanno, B.; Méndez-Alcaraz, J. M.; Nägele, G.; Klein, R.; Weber, R. J. Phys.: Condens. Matter 1991, 3, 4459.

(12) Wagner, N. J.; Krause, R.; Rennie, A. R.; D’Aguanno, B.; Goodwin, J. J. Chem. Phys. 1991, 95, 494.

(13) Zackrisson, M.; Stradner, A.; Schurtenberger, P.; Bergenholtz, J. Langmuir 2005, 21, 10835.

(14) Lebowitz, J. L. Phys. Rev. A 1964, 133, 895

(15) Baxter, R. J. J. Chem. Phys. 1970, 52, 4559.

(16) Vrij, A. J. Chem. Phys. 1979, 71, 3267.

(17) Blum, L.; Stell, G. J. Chem. Phys. 1979, 71, 42; 1980, 72, 2212

(18) Senatore, G.; Blum, L. J. Phys. Chem. 1985, 89, 2676.

(19) Robertus, C.; Philipse, W. H.; Joosten, J. G. H.; Levine, Y. K. J. Chem. Phys. 1989, 90, 4482.

(20) Gazzillo, D.; Giacometti, A.; Carsughi, F. J. Chem. Phys. 1997, $107,10141$.

(21) Ginoza, M.; Yasutomi, M. Phys. Rev. E 1999, 59, 2060.

(22) Carsughi, F.; Giacometti, A.; Gazzillo, D. Comput. Phys. Commun. 2000, 133,66

(23) Kotlarchyk, M.; Chen, S.-H. J. Chem. Phys. 1983, 79, 2461.
(24) Hayter, J. B. In Physics of amphiphiles: Micelles, vesicles and microemulsions; Degiorgio, V., Corti, M., Eds.; North-Holland: Amsterdam, 1985; pp 59-93.

(25) Pedersen, J. S. J. Appl. Crystallogr. 1994, 27, 595

(26) Griffith, W. L.; Triolo, R.; Compere, A. L. Phys. Rev. A 1987, 35, 2200 .

(27) Frenkel, D.; Vos, R. J.; de Kruif, C. G.; Vrij, A. J. Chem. Phys. 1986, $84,4625$.

(28) Olsson, U.; Schurtenberger, P. Langmuir 1993, 9, 3389.

(29) Olsson, U.; Würz, U.; Strey, R. J. Phys. Chem. 1993, 97, 4535.

(30) Leaver, M. S.; Olsson, U.; Wennerström, H.; Strey, R.; Würz, U.

J. Chem. Soc., Faraday Trans. 1995, 91, 4269.

(31) Bagger-Jörgensen, H.; Olsson, U.; Mortensen, K. Langmuir 1997, 13, 1413 .

(32) Knaapila, M.; Svensson, C.; Barauskas, J.; Zackrisson, M.; Nielsen, S. S.; Toft, K. N.; Vestergaard, B.; Arleth, L.; Olsson, U.; Pedersen, J. S.; Cerenius, Y. J. Synchrotron Radiat., in press.

(33) Schulz, G. V. Z. Phys. Chem., Abt. B 1939, 43, 25

(34) Zimm, B. H. J. Chem. Phys. 1948, 16, 1099.

(35) Aragón, S. R.; Pecora, R. J. Chem. Phys. 1976, 64, 2395.

(36) Auvray, L.; de Gennes, P. G. Europhys. Lett. 1986, 2, 647.

(37) Hone, J. H. E.; Cosgrove, T.; Saphiannikova, M.; Obey, T. M.; Marshall, J. C.; Crowley, T. L. Langmuir 2002, 18, 855.

(38) Bartlett, P.; Ottewill, R. H. J. Chem. Phys. 1992, 96, 3306.

(39) Yan, Y. D.; Clarke, J. H. R. J. Chem. Phys. 1990, 93, 4501.

(40) van Beurten, P.; Vrij, A. J. Chem. Phys. 1981, 74, 2744.

(41) Pusey, P. N.; van Megen, W. J. Chem. Phys. 1984, 80, 3513.

(42) Daoud, M.; Cotton, J. P. J. Phys. (Paris) 1982, 43, 531.

(43) Förster, S.; Burger, C. Macromolecules 1998, 31, 879.

(44) Leaver, M. S.; Olsson, U. Langmuir 1994, 10, 3449.

(45) Bagger-Jörgensen, H.; Olsson, U.; Jönsson, B. J. Phys. Chem. B 1997, 101, 6504.

(46) Zackrisson, M.; Andersson, R.; Bergenholtz, J. Langmuir 2004, 20,3080 .

(47) Caponetti, E.; Floriano, M. A.; Dio, E. D.; Triolo, R. J. Appl. Crystallogr. 1993, 26, 612.

(48) Balogh, J.; Olsson, U.; Pedersen, J. S. J. Dispersion Sci. Technol. 2006, 27, 497.

(49) Balogh, J.; Olsson, U.; Pedersen, J. S. J. Phys. Chem. B 2007, 111, 682.

(50) Gradshteyn, I. S.; Ryzhik, I. M. Tables of integrals, series, and products; Academic Press: New York, 1980. 